\title{
Determinantes de la lealtad en la relación entre la Liga Antioqueña de Tenis de Campo y sus clientes *
}

\author{
Determinants of Loyalty in the Relationship Between Liga Antioqueña de Tenis
}

de Campo and its Customers

\author{
Oscar Eduardo Ávila-Rodríguez \\ Magíster en Gestión Organizacional, Instituto Tecnológico Metropolitano, \\ Medellín - Colombia, oscaravila274140@correo.itm.edu.co \\ Silvana Janeth Correa-Henao \\ Magíster en Ciencia, Tecnología e Innovación, Instituto Tecnológico Metropolitano, \\ Medellín - Colombia, silvanacorreaitm@gmail.com \\ Laura Cristina Henao-Colorado \\ Magíster en Administración, Instituto Tecnológico Metropolitano, \\ Medellín - Colombia, laurahenao@itm.edu.co \\ Héctor Alonso Monroy-Escudero \\ Magíster en Administración, Liga Antioqueña de Tenis de Campo, \\ Medellín - Colombia, wgiraldo@unillanos.edu.co \\ Jorge Iván Brand-Ortiz \\ PhD en Gestión de la Tecnología y la Innovación, Instituto Tecnológico Metropolitano, \\ Medellín - Colombia, directorlat@une.net.co
}

\begin{abstract}
Cómo citar / How to cite
Ávila-Rodríguez, O. E., Correa-Henao, S. J., Henao-Colorado, L. C., Monroy-Escudero, H. A., Brand-Ortiz, J. I. (2021). Determinantes de la lealtad en la relación entre la Liga Antioqueña de Tenis de Campo y sus clientes. Revista CEA, v. 7, n. 15, e1811. https://doi.org/10.22430/24223182.1811
\end{abstract}

Recibido: 6 de abril de 2021

Aceptado: 3 de junio de 2021

\footnotetext{
* Este artículo se deriva del proyecto titulado «Factores determinantes de la lealtad de los usuarios de la Liga Antioqueña de Tenis para la construcción de escenarios estratégicos futuros» y ha sido financiado con recursos del Instituto Tecnológico Metropolitano.
} 


\title{
Resumen
}

Las organizaciones deportivas deben tomar decisiones acertadas en el ámbito del marketing, por lo que es necesario que dichas decisiones se basen en estudios que permitan identificar las características de las relaciones que mantienen con sus clientes, esto con el fin de que estas sean duraderas en el tiempo. En la literatura mundial se evidencian estudios que relacionan el entorno empresarial con el deportivo, pero pocos han estudiado este tipo de relación utilizando en su conjunto los constructos de satisfacción, valor percibido, confianza, compromiso y lealtad. Por lo tanto, este artículo pretende analizar los determinantes de la lealtad en la relación de la Liga Antioqueña de Tenis de Campo con sus clientes. La investigación se llevó a cabo a través de una metodología mixta que incluye dos fases: una fase exploratoria para comprender el problema de investigación y establecer las hipótesis, y otra descriptiva, de corte transversal simple, con análisis mediante un modelo de ecuaciones estructurales, bajo el enfoque de mínimos cuadrados parciales. El estudio arrojó una confirmación total de las hipótesis planteadas para el modelo, evidenciando los determinantes de la lealtad indicados por el marketing relacional en el contexto deportivo, específicamente en el de la Liga Antioqueña de Tenis de Campo y sus usuarios, encontrando que los determinantes directos de la lealtad, y que presentan un efecto positivo, son la satisfacción, la confianza y el compromiso.

Palabras clave: conocimiento del cliente, determinantes de lealtad, marketing relacional, organizaciones deportivas, ligas deportivas.

Clasificación JEL: C12, C39, C69, L31, L83, M31.

\section{Highlights}

- Los determinantes directos de la lealtad de marca en el contexto deportivo son la confianza, el compromiso y la satisfacción de los clientes.

- El marketing relacional es una estrategia fundamental que ayuda a construir relaciones a largo plazo con el cliente.

- El rol de los trabajadores es importante para construir relaciones rentables con los clientes.

\begin{abstract}
In order to have long-lasting relationships with their customers, sports organizations should make good marketing decisions based on studies that identify the characteristics of such relationships. In the literature, some studies have related the corporate environment to its sports counterpart, but few of them have investigated this kind of relationship using the set of constructs composed of satisfaction, perceived value, trust, commitment, and loyalty. Therefore, this paper analyzes the determinants of loyalty in the relationship between Liga Antioqueña de Tenis de Campo and its customers. This study adopted a mixed-methods approach divided into two stages: (1) exploration to understand the research problem and formulate the hypotheses and (2) description using a simple cross-sectional design with an analysis by Partial Least Squares Structural Equation Modeling (PLSSEM). The results confirmed all the hypotheses proposed in the model, which included the determinants of loyalty suggested by relationship marketing in the sports context. This study found that, in the relationship between Liga Antioqueña de Tenis de Campo and its users, satisfaction, trust, and commitment are the direct determinants of loyalty and have a positive effect on it.
\end{abstract}


Keywords: Customer knowledge, determinants of loyalty, relationship marketing, sports organizations, sports leagues.

JEL classification: C12, C39, C69, L31, L83, M31.

\section{Highlights}

- In the sports context, the direct determinants of brand loyalty are trust, commitment, and customer satisfaction.

- Relationship marketing is an essential strategy that helps to build long-term relationships with customers.

- Employees play an important role in establishing profitable relationships with customers.

\section{INTRODUCCIÓN}

El marketing ha tenido una evolución, pasando de tener un enfoque en transacciones, a basarse en generar relaciones de largo plazo (Vivek et al., 2009), donde la prioridad es conocer mejor a los clientes y entender cuál es la base de su valor como medio de diferenciación (Grönroos, 1997; Ardley et al., 2020). Dado lo anterior, varias investigaciones demuestran que para cultivar dichas relaciones y hacerlas perdurables en el tiempo, las empresas - de cualquier sector - deben entender y acercarse más a los clientes desde lo que estos perciben, quieren y sienten.

En Colombia, dentro del sector deportivo se tienen diferentes actores, tanto públicos como privados, que en el ámbito nacional, departamental y local forman todo el sistema nacional del deporte. En este caso, la entidad seleccionada es una organización deportiva sin ánimo de lucro (ODSL), las cuales son altamente dependientes de sus grupos de interés, quienes tienen intereses económicos y requieren de resultados (Ivašković, 2019), tienden a ser pequeñas y, a menudo, tienen niveles variables de capacidad de recursos (Brownlee et al., 2005), siendo necesario que promuevan actividades socialmente útiles para la comunidad en materia deportiva.

En el caso específico de la Liga Antioqueña de Tenis (LAT), fundada en 1938 como institución privada sin ánimo de lucro, se tiene que su principal finalidad es la difusión y promoción del deporte a través de diversos programas, y su compromiso con los usuarios es prepararlos para ganar (LAT, 2019). Sin embargo, para la LAT competir año tras año con entidades que ofrecen servicios gratuitos de tenis ha implicado un reto, dada la disminución de sus usuarios. En la disciplina del tenis de campo, si bien cuenta con clubes adscritos, el gran peso que tienen los usuarios de competencias, práctica libre y formación (usuarios naturales), es considerable. Para 2017 contaban con 970 usuarios, los cuales han ido disminuyendo, contando actualmente con aproximadamente 600 (LAT, 2019).

Además, la estructura de la LAT está definida desde los miembros que se asocian o afilian a ellas, y su objetivo es atraer más personas y socios con el tiempo para poder sostenerse y crecer (Winand et al., 2013), siendo, por tanto, esencial para ellos, indagar sobre la calidad que perciben sus miembros de los servicios que se están ofreciendo alrededor del tenis y cómo fidelizarlos para que no elijan los mismos servicios con otras entidades. De acuerdo con Winand et al. (2013), en las ODSL, el número creciente de proveedores de deportes comerciales son importantes para implementar nuevos 
servicios que retengan y sean atractivos para los miembros en su trabajo, necesitando, por tanto, adquirir nuevos recursos.

El artículo está distribuido por secciones. En primer lugar, se presenta el desarrollo, constituido por los elementos teóricos y el modelo de hipótesis; luego se encontrará la metodología llevada a cabo; posteriormente se hallan los resultados de la investigación, seguidos de la discusión y análisis; finalmente, se ubican las conclusiones.

\section{MARCO TEÓRICO}

El marketing ha tenido una evolución desde un enfoque basado en producir y lograr transacciones económicas con los clientes hasta una gestión desde el servicio, implicando que hoy en día se hable de marketing relacional, o basado en las relaciones con los clientes (Zeithaml et al., 2009; Nuryakin y Ardyan, 2018). El marketing relacional es definido como el proceso de identificar, establecer, mantener, mejorar, y cuando sea necesario, terminar las relaciones con los clientes y otros stakeholders de forma rentable, de tal manera que se cumplan los objetivos de las partes interesadas (Grönroos, 1997). Es así como se convierte a los clientes en el centro de cualquier estrategia que se quiera implementar (Mogaji et al., 2021; Bilovodska, 2017).

Para las empresas es importante retener a sus clientes en el largo plazo, siendo una necesidad inminente lograr construir relaciones duraderas donde se involucran factores como la confianza (Akbar y Parvez, 2009) y el compromiso para despertar ese deseo de involucramiento con la organización (Chaudhuri y Holbrook, 2001; Čater y Čater, 2010). Además, y yendo más allá, aparece el engagement como la intensidad de la participación de un individuo y su conexión con las ofertas de una organización y su involucramiento con las actividades que ésta realiza, logrado desde una buena experiencia de servicio (Brodie et al., 2011), siendo este factor, por tanto, fundamental como antecedente de la lealtad (Zainudin et al., 2019).

Asimismo, en el marco del marketing relacional, todos los factores anteriores son considerados antecedentes de la lealtad (Melnyk et al., 2009). Para el sector deportivo esto no es ajeno, dado que sus usuarios se caracterizan por tener elevados niveles de exigencia para lograr satisfacer sus necesidades en cuanto a resultados deportivos en el corto plazo; es decir, cada vez los socios son mucho más exigentes y tienen menos paciencia en satisfacer sus expectativas, y es por esto que las entidades deportivas están obligadas a llevar un seguimiento continuado de su calidad en los servicios (Nogales González, 2006).

Es por ello que se recomienda a los gerentes deportivos que se preocupen no solo por identificar lo que satisface a los consumidores para ganar su lealtad, sino también el punto de partida en el camino hacia la satisfacción (Pedragosa y Correia, 2009), como lo es el valor percibido (Clemes et al., 2011). Sumado a esto, se ha comprobado también la relación existente de los factores ya mencionados con la confianza, porque se considera factor relevante para la gestión deportiva, al orientar los ajustes en dimensiones específicas en pro de mejorar la calidad del servicio, conducir a la satisfacción y por tanto a una preferencia por el servicio en el largo plazo (Schijns et al., 2016). 


\section{Calidad de la relación}

Paek et al. (2020) definen la calidad de la relación en la industria deportiva como una evaluación general de fuerzas, siendo un constructo multidimensional que se caracteriza por elementos distintivos como la confianza y el compromiso, entre otros, los cuales, a su vez, han sido reconocidos como importantes para la calidad de las conexiones entre los usuarios y las organizaciones deportivas. Partiendo de esta definición, y bajo lo establecido por estos autores, se tiene que la confianza es la creencia de que alguien (otro individuo, empleados, empleadores, vendedores, otros clientes) actuará de manera transparente por un tercero. Asimismo, el compromiso es definido como la idea que tienen las personas de mantener una relación con un proveedor de servicios, mientras que al constructo se considera como la fuerza que capta la relación con un proveedor, el cual refleja el grado en que los clientes desean preservar esa relación más allá de transacciones funcionales, pudiendo estimular acciones cooperativas por parte de los clientes. Así, la calidad de la relación incluye la evaluación general que se tiene de una organización, donde se involucran constructos como la confianza y el compromiso (Gremler et al., 2020), y factores adicionales importantes como la satisfacción y la lealtad. La satisfacción, según la teoría de la desconfirmación, es el grado en que el rendimiento de un servicio excede, iguala o alcanza las expectativas de un individuo (Hyun, y Jordan, 2020), y se define como los sentimientos positivos luego del uso de un servicio. En el contexto del deporte, la satisfacción del espectador refleja una respuesta placentera respecto a servicios deportivos (Phonthanukitithaworn y Sellitto, 2018). Por último, la lealtad es una variable resultado de la primaria experiencia del cliente, y se define como la actitud o comportamiento de mantener una relación en el largo plazo con una organización (Pekovic y Rolland, 2020), incluyendo así, el deseo de permanecer en una relación y considerar a la organización como la primera en preferencia (Gremler et al., 2020).

\section{Satisfacción}

La satisfacción del cliente se define como los sentimientos de placer o decepción de una persona que resultan de comparar el desempeño o resultado percibido de un servicio con sus expectativas (Kotler y Keller, 2009). De igual forma, Birim et al. (2016) consideran que la satisfacción es uno de los principales constructos de interés en la investigación académica. Churchill y Surprenant (1982) definieron la satisfacción como un resultado importante de la actividad de marketing que sirve para vincular los procesos que culminan en la compra y el consumo con fenómenos posteriores a esa adquisición, como el cambio de actitud, la compra repetida y la lealtad a la marca. Si está satisfecho con un producto o servicio, un cliente puede comprar nuevamente (Birim et al., 2016). Por el contrario, si un cliente no está satisfecho, la probabilidad de recompra es menor (Matsuoka et al., 2003).

La satisfacción de los usuarios, generalmente, se basa en la noción de que una empresa debe satisfacer a sus clientes para ser sostenible y rentable (Farooq y Radovic-Markovic, 2016; Izogo y Ogba, 2015; Radovic-Markovic et al., 2017). Según Farooq et al. (2018), la satisfacción del cliente se define como sentimientos positivos posteriores al consumo. En lugar de explorar los resultados cognitivos, la satisfacción del cliente se considera una medida efectiva de la utilidad de un producto o servicio utilizado por los clientes (Berezina et al., 2012). 


\section{Compromiso}

El compromiso del consumidor se considera un componente fundamental que juega un papel clave en la gestión de la comunicación con el cliente en las relaciones de marketing a largo plazo (Fullerton, 2003; Gustafsson et al., 2005; Hess y Story, 2005; Hunt et al., 2006).

El compromiso refleja una postura de continuidad de la relación entre socios de intercambio (Dwyer et al., 1987). Por su parte, Moorman et al. (1992) lo describen como el deseo de un cliente de mantener una relación valorada a fin de garantizar los máximos esfuerzos para sostenerla. Por lo tanto, el compromiso implica la voluntad del cliente de hacer sacrificios para lograr ganancias a largo plazo (Khodabandeh y Lindh, 2020), y revela el apego del individuo a la marca, lo cual lo vuelve más indulgente en caso de problemas relacionados con el producto (por ejemplo, falla) y menos sensible a las actividades de marketing de la competencia (Hess y Story, 2005; Story y Hess, 2010).

El compromiso es una de las variables más importantes en la creación de relaciones a largo plazo dentro de un mercado. Esto se debe a que los clientes sienten que tienen un vínculo fuerte con la empresa, y este sentimiento juega un papel importante en el mantenimiento de las relaciones con los clientes (Quero y Ventura, 2015). Según Wu y Ko (2013), el compromiso con una marca o producto favorable se forma cuando la intención de compra del consumidor es positiva, lo que lleva a una compra real.

\section{Confianza}

De acuerdo con Čater y Čater (2010), la confianza se considera como una creencia de que en una relación algún actor procederá en el mejor interés del otro y tendrá la disposición de confiar en la integridad y la capacidad de éste Garbarino y Johnson, 1999; Palmatier et al., 2007; Peña García, 2104; Taylor y Hunter, 2003).

Por otra parte, la confianza se considera como la expectativa que tiene el cliente de que el proveedor de servicios cumplirá sus promesas (Sirdeshmukh et al., 2002), siendo relevante en momentos de incertidumbre (Chaudhuri y Holbrook, 2001), porque reduce la ansiedad y el riesgo, aumenta la fe en el proveedor y da conocimiento sobre qué esperar (Chiou y Droge, 2006). De igual forma, se estima a la confianza como uno de los antecedentes más relevantes de las relaciones estables y de colaboración que ofrece a los clientes una garantía de resultados positivos para el presente y el futuro» (Akbar y Parvez, 2009).

La investigación académica ha acordado definir la confianza en torno a la idea de dependencia de la integridad, capacidad o carácter de una persona o cosa; es decir, el deseo de confiar en la integridad de un tercero con quien se ha establecido o se va a establecer un intercambio (Khodabandeh y Lindh,

2020) o, en la práctica, con la creencia de una persona en la promesa de otro (Khodabandeh y Lindh, 2020).

Si existe confianza, los individuos no son propensos a creer que los vendedores se comportarán de manera oportunista (Gefen et al., 2003), y esto refuerza, a su vez, el paradigma relacional que alimenta el ciclo de marketing que sustenta buena parte de la investigación académica en este campo en años recientes. 


\section{Lealtad}

La lealtad del cliente es probablemente una de las mejores medidas de éxito en cualquier organización (Nyadzayo y Khajehzadeh, 2016). El desarrollo, el mantenimiento y la mejora de la lealtad de los usuarios siguen siendo un foco central de la mayoría de las actividades de marketing de las empresas (Dick y Basu, 1994). De igual forma, la lealtad del cliente generalmente se interpreta en términos de comportamiento de compra (Payne y Frow, 2013) y se conoce principalmente como comportamientos -futuros- o intenciones de comportamiento (Avourdiadou y Theodorakis, 2014; Howat y Assaker, 2013; Murray y Howat, 2002).

Aumentar el número de usuarios leales y mantenerlos con una organización es fundamental para el éxito a largo plazo de la empresa (TaghiPourian y Bakhsh, 2015). Un cliente altamente leal compra repetidamente un producto/servicio y posee un sentido positivo de lealtad actitudinal hacia la marca (Han y Hyun, 2018). Dado esto, generar y mantener la lealtad del usuario ha sido un tema importante entre los profesionales de la industria del marketing (Bowen y Chen McCain, 2015; Chua et al., 2015).

\section{Modelo de Hipótesis}

Los constructos identificados como determinantes de la lealtad para este trabajo fueron validados mediante un modelo de hipótesis construido con base en la interacción que tienen estos dentro de la lógica del marketing relacional. El modelo se representa en la Figura 1.

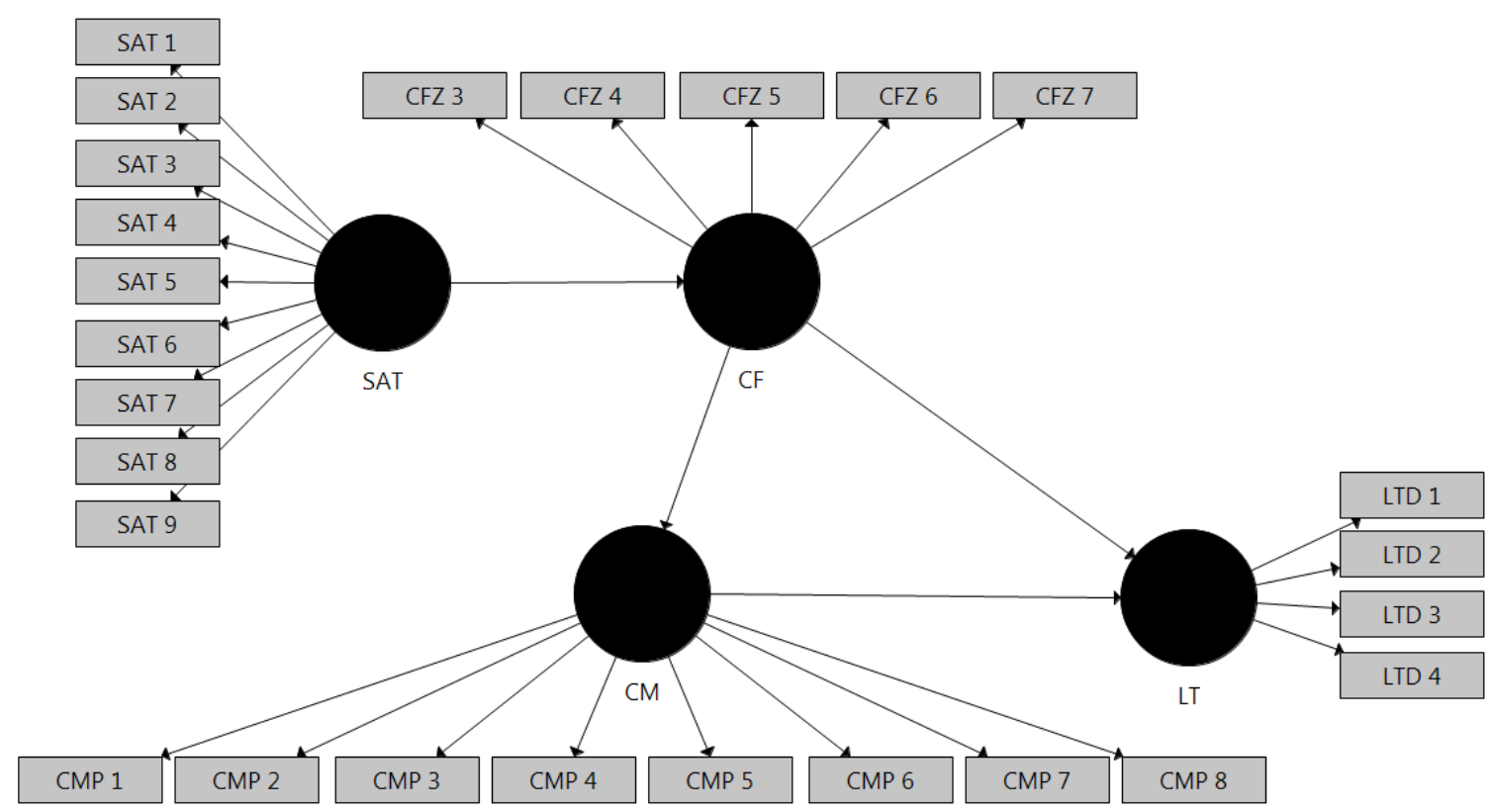

Figura 1. Modelo de hipótesis para la relación LAT y usuarios

Figure 1. Hypothesis model of the relationship between the LAT and its users Fuente: elaboración propia.

En la literatura se encontró que diversos autores toman la satisfacción como constructo influenciador de la confianza (Garbarino y Johnson, 1999; Oliver, 1999; Parasuraman et al., 1985), ya que una 
acumulación de satisfacción en la experiencia del servicio permite que el cliente genere confianza en la relación. En el contexto deportivo, también se confirma la relación (Filo et al., 2008).

H1: la satisfacción de los usuarios de la LAT influye positiva y directamente en la confianza de dichos usuarios.

La confianza es considerada un constructo importante para la generación de relaciones de largo plazo (Jau-Chuan et al., 2011). Morgan y Hunt (1994) consideran la confianza y el compromiso como elementos esenciales de la construcción de las relaciones. Asimismo, se ha abordado la confianza como influenciador del compromiso (Morgan y Hunt, 1994; Moorman et al., 1992; Achrol, 1991; Hrebiniak, 1974).

De igual forma, se ha encontrado que la confianza se considera antecedente de la lealtad (Morgan y Hunt, 1994; Moorman et al., 1993; Palmatier et al., 2012) y en el contexto del deporte también se refleja relevancia entre la relación de estos dos tópicos (Alhaddad 2015). Sin embargo, en la literatura del deporte la confianza es importante para generar lealtad, pero no es indispensable por sí sola (Filo et al., 2008), por lo que se encuentran estudios como el de Seyed Javadein et al. (2008), donde genera efectos indirectos a través del compromiso.

H2: la confianza de los usuarios de la LAT influye positiva y directamente en el compromiso de dichos usuarios.

H3: la confianza de los usuarios de la LAT influye positiva y directamente en la lealtad de dichos usuarios.

El compromiso en la literatura ha sido identificado como influenciador de la lealtad (Garbarino y Johnson, 1999; Morgan y Hunt, 1994; Moorman et al., 1992), ya que ante un alto grado de compromiso en la relación es muy factible que se genere la lealtad.

H4: el compromiso de los usuarios de la LAT influye positiva y directamente en la lealtad de dichos usuarios.

\section{METOdOLOGÍA}

La investigación se realizó a través de una metodología mixta, teniendo un componente exploratorio y uno descriptivo para el análisis de los determinantes de los antecedentes de la lealtad en la relación que tiene la LAT con sus clientes.

En primer lugar, se realizó una fase exploratoria donde por medio de consulta en fuentes secundarias, se construyó el estado del arte necesario para la comprensión del problema de investigación y el planteamiento de las hipótesis para el modelo relacional. Se evidenciaron investigaciones a nivel mundial, latinoamericano y nacional sobre los constructos satisfacción, confianza, compromiso y lealtad.

En segundo lugar, se realizó una fase descriptiva de corte transversal simple, donde se aplicó una encuesta autoadministrada a los usuarios afiliados a la LAT, construida con escalas estructuradas y 
validadas en la literatura, las cuales fueron traducidas y adaptadas al contexto estudiado (deportes) para garantizar el entendimiento de las mismas.

En este punto, la estrategia de implementación de dicha encuesta consistió en asistir a las instalaciones de la LAT para aplicar la prueba de manera presencial con los diferentes usuarios deportivos de la organización. Una vez transcurrió un tiempo considerable de aplicación de encuestas en esta sede, se procedió a asistir a uno de los eventos deportivos programados en el calendario de la LAT para recolectar más información que permitiera fortalecer el número total de muestras para el estudio. De igual forma, se procedió con el envío de las encuestas de forma virtual a través de un formulario que fue respondido por los diferentes clientes de la organización.

Una vez finalizada la etapa anterior, se procedió a tabular y registrar en Excel los datos adquiridos, descartando las encuestas que tuvieran errores (doble marcación, tachones, enmendaduras, respuestas incompletas, entre otros), obteniendo el total del tamaño de muestreo: 174 encuestas con un nivel de confianza del $97 \%$ y un error muestral del $5 \%$. Este archivo fue incorporado en el programa SmartPLS, y a partir de su ingreso, se procedió con la etapa del tratamiento de datos.

No obstante, dada la dificultad para contar con el tiempo de los usuarios de la LAT, el muestreo fue no probabilístico a conveniencia y juicio de los investigadores, bajo el criterio de lugar de entrenamiento, clases y competencias (sede Tenis de Campo - Estadio y Clubes Sociales donde se lleven a cabo los torneos y competencias); además, se requirió que sus usuarios tuvieran relación con la LAT y accedieran a los servicios de competencia, formación y práctica libre. En la Tabla 1 se relaciona la ficha técnica del estudio.

Tabla 1. Ficha técnica del estudio

Table 1. Technical specifications of the survey

\begin{tabular}{cc}
\hline Unidad muestral & Clientes de competencia, formación y práctica libre \\
\hline Ámbito de estudio & Liga Antioqueña de Tenis de Campo \\
\hline Método de recogida de información & Encuesta física y virtual \\
\hline Procedimiento de muestreo & $\begin{array}{c}\text { No probabilístico a conveniencia y juicio de los } \\
\text { investigadores }\end{array}$ \\
\hline Tamaño muestral & $\mathrm{n}=174$ \\
\hline Nivel de confianza & $97 \%$ \\
\hline Error muestral & $5 \%$ \\
\hline Fecha del trabajo de campo & Junio a agosto de 2019
\end{tabular}

De acuerdo con la Tabla 1, a pesar de ser una muestra no probabilística, se considera representativa dado que garantiza las características que se quieren observar en la población, quedando expresadas apropiadamente en la muestra (usuarios de los tres servicios ofrecidos por la Liga). Igualmente, los datos obtenidos por los 174 usuarios de la LAT se repartieron de la siguiente forma: i) formación deportiva (47\%); ii) práctica (18\%); y iii) torneos y competencias (33\%). Los anteriores porcentajes están dados de acuerdo con el total de usuarios con los que cuenta la LAT en sus diferentes servicios (590 usuarios).

El instrumento se compone de 32 ítems: 9 ítems para medir el constructo satisfacción (Oliver, 1980; Westbrook y Oliver, 1981; Voss et al., 1998; De Wulf, et al., 2001); 7 para medir el factor confianza 
(Anderson y Weitz, 1992; Ganesan, 1994; Kumar et al., 1995; Chaudhuri y Holbrook, 2001; Lashley y Morrison, 2003); 8 para medir el compromiso (Anderson y Weitz, 1992; Price y Arnould, 1999; Tax et al., 1998); y por último, 8 variables de medida para el constructo lealtad (Zeithaml et al., 1996; Bell et al., 2005; Auh et al., 2007). Las variables fueron adaptadas al contexto deportivo y se midieron utilizando la escala de Likert de cinco puntos que varió entre 1=muy en desacuerdo y 5=muy de acuerdo.

Finalmente, los datos obtenidos fueron analizados mediante el método de modelo de ecuaciones estructurales, bajo el enfoque mínimos cuadrados parciales PLS-SEM) de Ringle et al. (2015) para comprobar las hipótesis planteadas para el modelo relacional y así analizar los determinantes de la lealtad por parte de los usuarios de la LAT.

\section{RESULTADOS}

\section{Fiabilidad y Validez}

En primera instancia, se hace énfasis en el instrumento de medida para constructos reflexivos (Hair et al., 2017). En esa medida se presentan valores aceptables del alfa de Cronbach -CA- y fiabilidad compuesta -CR- para todos los constructos reflexivos, lo que demuestra la consistencia interna y la confiabilidad del instrumento aplicado en el estudio, así como se expone en la Tabla 2. Adicionalmente, los valores de la varianza media extraída (AVE, por sus siglas en inglés) de los constructos reflexivos son mayores que 0.5 , y todos los tamaños de carga, además de significativos, han sido mayores de 0.6. Estos valores proporcionan evidencia de cumplimiento de la validez convergente; es decir, los elementos han medido los constructos respectivos con firmeza, evidenciándolo en la Tabla 2.

Para este caso se comprueba la consistencia interna del modelo, teniendo en cuenta un CA mayor a 0.7 (Nunnally y Bernstein, 1994), una CR mayor a 0.7 (Fornell y Larcker, 1981) y una validez convergente con una AVE mayor a 0.5 (Fornell y Larcker, 1981) cargas mayores a 0.6 y significativas con $\mathrm{p}<0001$ (Bagozzi y Yi, 1988).

En segundo lugar, se realizó una comprobación de la validez discriminante del modelo, descrita en la Tabla 2. Para esta medida, determinada por Fornell y Larcker (1981), Hair et al. (2017) sugieren proceder con el análisis de los valores diagonales como las raíces cuadradas de AVE y luego revisar si estas variaciones han sido mayores que la correlación cuadrática más alta de las construcciones presentadas debajo de la diagonal, demostrando así la validez discriminante. Se cumple el criterio para cada uno de los constructos (ver Tabla 3). 
Oscar Eduardo Ávila-Rodríguez / Silvana Janeth Correa-Henao / Laura Cristina Henao-Colorado Héctor Alonso Monroy-Escudero / Jorge Iván Brand-Ortiz

Tabla 2. Validez convergente y fiabilidad

Table 2. Convergent validity and reliability

\begin{tabular}{|c|c|c|c|c|c|}
\hline Dimensión & Ítems & Cargas & $\mathrm{CA}$ & CR & AVE \\
\hline \multirow{5}{*}{ Confianza } & $\mathrm{C} 1$ & $0.930 * * *$ & \multirow{5}{*}{0.971} & \multirow{5}{*}{0.977} & \multirow{5}{*}{0.895} \\
\hline & $\mathrm{C} 2$ & $0.927^{* * *}$ & & & \\
\hline & $\mathrm{C3}$ & $0.952^{* * *}$ & & & \\
\hline & $\mathrm{C} 4$ & $0.965^{* * *}$ & & & \\
\hline & $\mathrm{C5}$ & $0.956^{* * *}$ & & & \\
\hline \multirow{8}{*}{ Compromiso } & CM1 & $0.840 * * *$ & \multirow{8}{*}{0.965} & \multirow{8}{*}{0.971} & \multirow{8}{*}{0.805} \\
\hline & $\mathrm{CM} 2$ & $0.897 * * *$ & & & \\
\hline & $\mathrm{CM} 3$ & $0.880 * * *$ & & & \\
\hline & $\mathrm{CM} 4$ & $0.939 * * *$ & & & \\
\hline & CM5 & $0.904^{* * *}$ & & & \\
\hline & CM6 & $0.925^{* * *}$ & & & \\
\hline & CM7 & $0.880 * * *$ & & & \\
\hline & $\mathrm{CM} 8$ & $0.911^{* * *}$ & & & \\
\hline \multirow{4}{*}{ Lealtad } & $\mathrm{L} 1$ & $0.924 * * *$ & \multirow{4}{*}{0.955} & \multirow{4}{*}{0.968} & \multirow{4}{*}{0.882} \\
\hline & L2 & $0.963 * * *$ & & & \\
\hline & L3 & $0.949 * * *$ & & & \\
\hline & $L 4$ & $0.920 * * *$ & & & \\
\hline \multirow{9}{*}{ Satisfacción } & S1 & $0.934 * * *$ & \multirow{9}{*}{0.981} & \multirow{9}{*}{0.984} & \multirow{9}{*}{0.870} \\
\hline & S2 & $0.937 * * *$ & & & \\
\hline & S3 & $0.943^{* * *}$ & & & \\
\hline & S4 & $0.915^{* * *}$ & & & \\
\hline & S5 & $0.935 * * *$ & & & \\
\hline & S6 & $0.924 * * *$ & & & \\
\hline & S7 & $0.916 * * *$ & & & \\
\hline & S8 & $0.943 * * *$ & & & \\
\hline & S9 & $0.945^{* * *}$ & & & \\
\hline
\end{tabular}

Fuente: elaboración propia a partir de cálculos arrojados en software SmartPLS 3.0.

Tabla 3. Validez discriminante del modelo

Table 3. Discriminant validity of the model

\begin{tabular}{ccccc}
\hline & CF & CM & LT & ST \\
\hline Confianza & 0.946 & & & \\
\hline Compromiso & 0.835 & 0.897 & & \\
\hline Lealtad & 0.810 & 0.897 & 0.939 & \\
\hline Satisfacción & 0.858 & 0.847 & 0.829 & 0.933 \\
\hline
\end{tabular}

Fuente: elaboración propia a partir de cálculos arrojados en software SmartPLS 3.0.

Se evidencia que el modelo presenta una validez discriminante, teniendo en cuenta que el cuadrado de AVE, mostrada en la diagonal de la matriz, presenta valores mayores a 0.7 (Chin, 1998).

En tercer lugar, se realizó la evaluación de la capacidad predictiva del modelo, teniendo en cuenta el $\mathrm{R}^{2}$, el cual indica el porcentaje de variación de la variable de respuesta que explica su relación con una o más variables predictoras (Aldás, 2017). Los resultados calculados por el software SmartPLS 3.0 
evidencian que los constructos confianza $\left(R^{2}=0.736\right)$, compromiso $\left(R^{2}=0.698\right)$ y lealtad $\left(R^{2}=0.817\right)$, son indicadores relevantes, según Hair et al. (2014).

\section{Análisis confirmatorio}

Se llevaron a cabo las pruebas de hipótesis calculadas por medio del bootstrapping para obtener los coeficientes de las trayectorias y sus valores $t$ mostrando como resultado que las cuatro hipótesis planteadas fueron significativas. Es así que se confirman las relaciones positivas y directas de: satisfacción-confianza H1 ( $\mathrm{t}=8.858$; p.value $<0.001$ ), confianza-compromiso $\mathrm{H} 2$ ( $\mathrm{t}=0.835$; p.value $<0.001$ ), confianza-lealtad H3 ( $\mathrm{t}=0.200$; p.value $<0.01$ ) y compromiso-lealtad $\mathrm{H} 4$ ( $\mathrm{t}=0.730$; p.value $<0.001$ ). En esa medida se realiza el contraste de hipótesis, evidenciando su confirmación en la Tabla 4.

Tabla 4. Contraste de hipótesis

Table 4. Hypothesis testing

\begin{tabular}{ccccc}
\hline Hipótesis & Descripción & $\begin{array}{c}\text { Coeficiente de } \\
\text { trayectoria }\end{array}$ & Valor $t$ & Significancia \\
\hline $\mathrm{H} 1$ & Satisfacción-> Confianza & 0.858 & 34107 & $* * *$ \\
\hline $\mathrm{H} 2$ & Confianza-> Compromiso & 0.835 & 26392 & $* * *$ \\
\hline $\mathrm{H} 3$ & Confianza-> Lealtad & 0.200 & 2.708 & $* *$ \\
\hline $\mathrm{H} 4$ & Compromiso -> Lealtad & 0.730 & 9.818 & $* * *$ \\
\hline \multicolumn{5}{c}{ P<0.001***; $p<0.01^{* *}$} \\
Fuente: elaboración propia.
\end{tabular}

Se puede observar que las cuatro hipótesis fueron confirmadas, teniendo en cuenta el nivel de significancia presentado por los coeficientes de trayectoria. En la Figura 2 se muestran los resultados de manera gráfica.

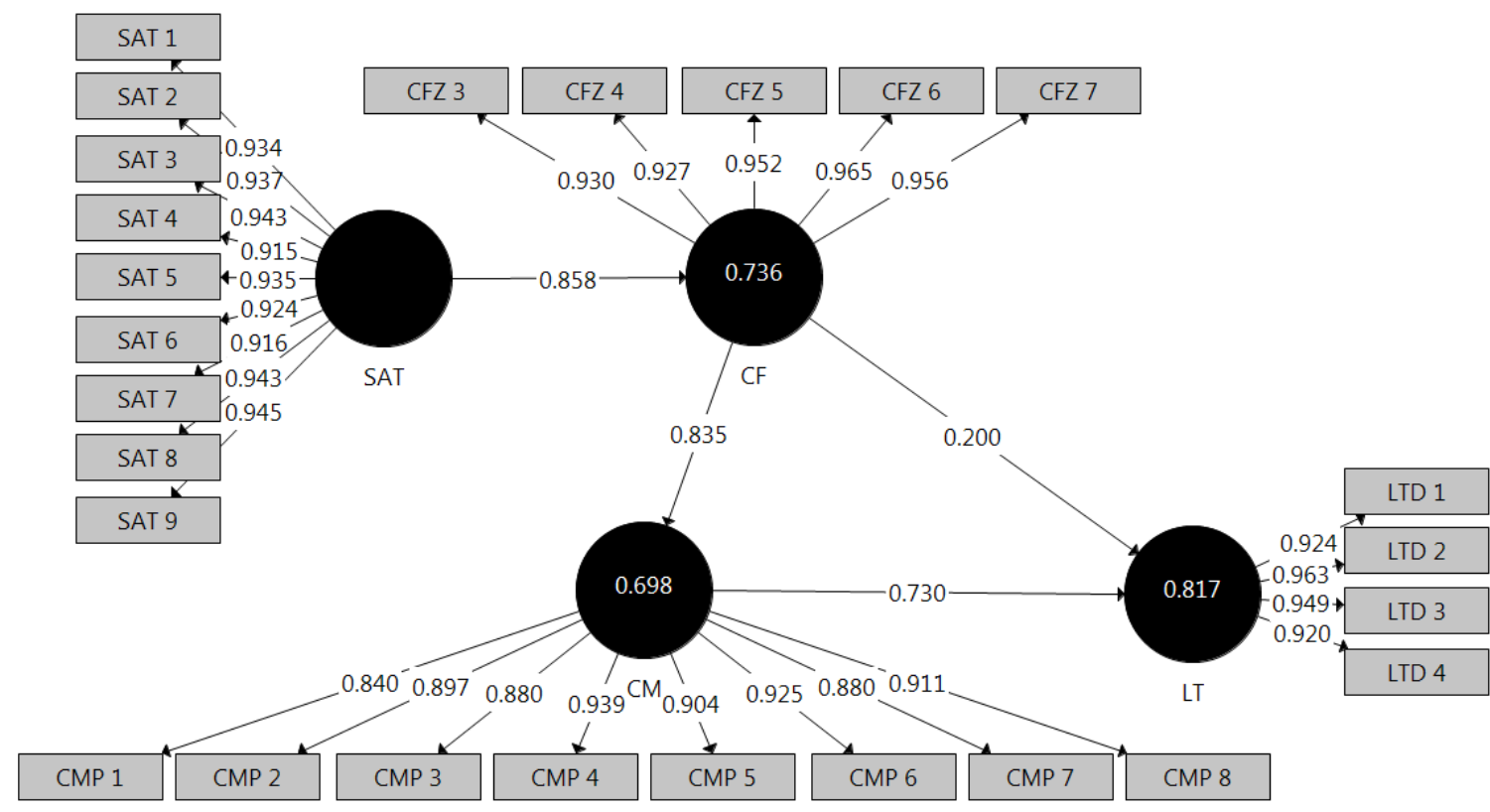

Figura 2. Resultados del modelo estructural

Figure 2. Results of the structural model

Fuente: elaboración propia a partir de resultados del software SmartPLS. 


\section{DISCUSIÓN}

Los resultados del modelo arrojan una confirmación total de las hipótesis planteadas para el modelo, evidenciando como los determinantes de la lealtad, indicados por el marketing relacional, lo son en el contexto deportivo, específicamente en el caso de la Liga Antioqueña de Tenis de Campo y sus usuarios.

La hipótesis 1 plantea que existe una relación directa y positiva entre la satisfacción de los usuarios de la LAT y su confianza. En términos generales se ha evidenciado que existe una satisfacción por parte de los usuarios, lo que permite desarrollar sentimientos de confianza hacia la LAT. Esta hipótesis es confirmada ( $\mathrm{H} 1$ : $\beta=0.858 ; \mathrm{p}=0.000$ ), por lo que se concluye que la satisfacción tiene un efecto positivo y significativo sobre la confianza en la LAT, coincidiendo con el trabajo de Seyed Javadein et al. (2008).

La hipótesis 2 establece que existe una relación directa y positiva entre la confianza de los usuarios de la LAT y su compromiso. En la literatura se muestra que la confianza es esencial para generar compromiso. Esta hipótesis es confirmada ( $\mathrm{H} 2$ : $\beta=0.835 ; \mathrm{p}=0.000)$, por lo que se concluye que la confianza tiene un efecto positivo y significativo sobre el compromiso en la LAT, en línea con Clark y Maher (2007) y Seyed Javadein et al. (2008).

La hipótesis 3 muestra que existe una relación directa y positiva entre la confianza de los usuarios de la LAT y su lealtad. En la literatura se evidencia que la confianza tiene un efecto sobre la generación de lealtad y las relaciones de largo plazo. Esta hipótesis es confirmada (H3: $\beta=0.200 ; p=0.007$ ), por lo que se concluye que la confianza tiene un efecto positivo y significativo sobre la lealtad en la LAT, de acuerdo con Filo et al. (2008).

La hipótesis 4 indica que existe una relación directa y positiva entre el compromiso de los usuarios de la LAT y su lealtad. En la literatura se encuentra que el compromiso es un paso importante para propiciar la lealtad y el establecimiento de relaciones de largo plazo. Esta hipótesis es confirmada ( $H 4$ : $\beta=0.730 ; p=0.000$ ), por lo que se concluye que el compromiso tiene un efecto positivo y significativo sobre la lealtad en la LAT, de acuerdo con Moreira y Silva (2015) y Seyed Javadein et al. (2008).

Con base en lo anterior, se puede decir que los determinantes directos de la lealtad, y que presentan un efecto positivo, son la confianza y el compromiso, siendo la confianza antecedida por la satisfacción, que es la base para la generación de la misma en el contexto de la relación entre la LAT y sus clientes.

En esa medida, esta investigación permite evidenciar los conceptos primordiales propuestos por Crosby et al. (1990); Moorman et al. (1993); Morgan y Hunt (1994); Doney y Cannon (1997); Dorsch et al. (1998); Priluck Grossman (1998); Crotts y Turner (1999); Price y Arnould (1999) y Ganesh et al. (2000), quienes aseveran que existe un elevado número de investigaciones dentro de la literatura del marketing relacional en torno al concepto de confianza como elemento primordial para mantener relaciones satisfactorias. 
De igual forma, autores como Parasuraman et al. (1985) sugieren que los clientes deberían ser capaces de confiar en los proveedores del servicio, sentirse seguros en el trato que mantienen con ellos, así como tener la certeza de que la información que les transmiten tendrá un carácter confidencial. En esa medida, para el caso específico de la Liga Antioqueña de Tenis de Campo, todas estas consideraciones son cruciales a la hora de obtener la fidelidad de sus clientes y contribuir positivamente a que la organización cuente con unos ingresos estables de ellos.

Es así que, de acuerdo con autores como Achrol (1991) y Moorman et al. (1992), un determinante esencial para la lealtad en los clientes reside en que una relación caracterizada por la existencia de confianza en la otra parte es positivamente valorada y sienta las bases para que el compromiso pueda estar presente.

Por último, es importante mencionar que, dentro de las implicaciones de los resultados obtenidos en la investigación, se concibe que las organizaciones, en este caso deportivas, deben preocuparse por tratar de desarrollar confianza en sus clientes, resultado compatible con los de estudios anteriores realizados en otros sectores de actividad (Garbarino y Johnson, 1999); Chaudhuri y Holbrook, 2001; Chiou y Droge, 2006). Es así que, en la medida que un cliente perciba el buen hacer de estas organizaciones, junto a su interés por satisfacer los deseos y necesidades del público objetivo, el clima de confianza entre ambas partes aumentará.

\section{CONCLUSIONES}

Este estudio permite demostrar los determinantes de la lealtad en el contexto de la relación entre la LAT y sus usuarios, lo cual representa un aporte para la construcción de la literatura del marketing y la gestión deportiva en países emergentes como Colombia.

Al comprobar la validez y la fiabilidad de las escalas de medida utilizadas para esta comprobación, se evidencia una pertinencia del uso de estas en futuras investigaciones alrededor del tema para analizar las relaciones entre estos constructos con otros que puedan generar preguntas de investigación.

Se puede confirmar que la confianza y la lealtad son antecedentes relevantes a la hora de generar lealtad en organizaciones deportivas como la LAT, lo que implica que los esfuerzos no se deben centrar solo en satisfacer a los usuarios sino en ir más allá en la generación de confianza y compromiso para el establecimiento de relaciones de largo plazo.

Todo esto permite concluir que la relación entre la LAT con sus usuarios es posible gestionarla teniendo en cuenta un enfoque relacional, permitiendo a las organizaciones deportivas estar a la vanguardia con lo que se espera de otro tipo de organizaciones donde el servicio es la base para la generación de valor.

Igualmente, el propósito buscado no debe ser exclusivamente lograr que los clientes vuelvan, en este caso, a la LAT para contratar servicios deportivos, sino que se debe promover una lealtad basada en las percepciones de sus clientes acerca de la prestación de los servicios. De este modo, el rol que tienen los trabajadores de la LAT es sumamente importante porque son los que interactúan 
directamente con los clientes, lo que permite evidenciar los beneficios de aplicar aspectos del marketing relacional.

Finalmente se debe manifestar el carácter colateral que tiene este estudio y su aplicación en un contexto limitado. De igual forma, el estudio de un mayor número de antecedentes de las variables expuestas en el trabajo compone una futura línea de investigación que posibilitará tener una visión más completa de la aplicación de la estrategia del marketing relacional en la distribución de servicios deportivos.

Las limitaciones del estudio que se presentaron partieron desde su diseño metodológico, teniendo en cuenta que las conclusiones generadas aplican para el contexto de la LAT, por lo que las relaciones encontradas pueden estar condicionadas por las características de los usuarios de esta liga.

\section{CONFLICTOS DE INTERÉS}

Los autores declaran que no presentan conflictos de interés financiero, profesional o personal que pueda influir de forma inapropiada en los resultados obtenidos o las interpretaciones propuestas.

\section{CONTRIBUCIÓN DE AUTORES}

Para el desarrollo de este proyecto todos los autores han realizado una contribución significativa especificada a continuación:

Oscar Eduardo Ávila-Rodríguez: conceptualización, desarrollo de la investigación y redacción.

Silvana Janeth Correa-Henao: conceptualización, diseño, desarrollo de la investigación y redacción.

Laura Cristina Henao-Colorado: conceptualización, diseño, desarrollo de la investigación, redacción y revisión del manuscrito.

Héctor Alonso Monroy-Escudero: desarrollo de la investigación.

Jorge Iván Brand-Ortiz: diseño y desarrollo de la investigación.

\section{REFERENCIAS}

Achrol, R. S. (1991). Evolution of the Marketing Organization: New Forms for Turbulent Environments. Journal of Marketing, v. 55, n. 4, 77-93. https://doi.org/10.1177/002224299105500406

Akbar, M. M., Parvez, N. (2009). Impact of services quality, trust and customer satisfaction on customer loyalty. ABAC Journal, v. 29, n. 1, 24-38. URL 
Aldás, J. (2017). Modelización estructural con PLS-SEM: Constructos de segundo orden. Universitat de València-Ivie.

Alhaddad, A. (2015). Perceived quality, brand image and brand trust as determinants of brand loyalty. Journal of Research in Business and Management, v. 3, n. 4, 1-8. URL

Anderson, E., Weitz, B. (1992). The use of pledges to build and sustain commitment in distribution channels. Journal of marketing research, v. 29, n. 1, 18-34.

https://doi.org/10.1177/002224379202900103

Ardley, B., McIntosh, E., McManus, J. (2020). From transactions to interactions: the value of cocreation processes within online brand consumer communities. Business Process Management Journal, v. 26, n. 4, 825-838. https://doi.org/10.1108/BPMJ-10-2019-0444

Auh, S., Bell, S. J., McLeod, C. S., Shih, E. (2007). Co-production and customer loyalty in financial services. Journal of retailing, v. 83, n. 3, 359-370.

https://doi.org/10.1016/j.jretai.2007.03.001

Avourdiadou, S., Theodorakis, N. D. (2014). The development of loyalty among novice and experencied customers of sport and fitness centres. Sport Management Review, v. 17, n. 4, 419-432. https://doi.org/10.1016/i.smr.2014.02.001

Bagozzi, R. P., Yi, Y. (1988). On the evaluation of structural equation models. Journal of the academy of marketing science, v. 16, n. 1, 74-94. https://doi.org/10.1007/BF02723327

Bell, S. J., Auh, S., Smalley, K. (2005). Customer relationship dynamics: service quality and customer loyalty in the context of varying levels of customer expertise and switching costs. Journal of the Academy of Marketing Science, v. 33, n. 2, 169-183.

https://doi.org/10.1177/0092070304269111

Berezina, K., Cobanoglu, C., Miller, B. L., Kwansa, F. A. (2012). The impact of information security breach on hotel guest perception of service quality, satisfaction, revisit intentions and word-of-mouth. International Journal Contemporany Hospital Management, v. 24, n. 7, 991-1010. https://doi.org/10.1108/09596111211258883

Bilovodska, O. A. (2017). Marketing distributive and sales policy: research of essence, role and importance. Marketing and management of innovations, n. 2, 85-97. http://doi.org/10.21272/mmi.2017.2-08

Birim, B., Anitsal, M. M., Anitsal, I. (2016). Perceived value, satisfaction, brand equity and behavioral intentions: Scale development for sports spectatorship in us college football. Academy of Strategic Management Journal, v. 15, n. 1, 1-11. URL 
Bowen, J. T., Chen McCain, S. -L. (2015). Transitioning loyalty programs: a commentary on the relationship between customer loyalty and customer satisfaction. International Journal Contemporany Hospitality Management, v. 27, n. 3, 415-430. https://doi.org/10.1108/IJCHM-07-2014-0368

Brodie, R., Hollebeek, L., Jurić, B., Ilić, A. (2011). Customer engagement: conceptual domain, fundamental propositions, and implications for research. Journal of Service Research, v. 14, n. 3, 252-271. https://doi.org/10.1177/1094670511411703

Brownlee, B., Gumulka, G., Barr, C., Lasby, D. (2005). Understanding the capacity of religious organizations: a Synthesis of Findings from the National Survey of Nonprofit and Voluntary Organizations and the National Survey of Giving, Volunteering and Participating. Imagine Canada.

Čater, T., Čater, B. (2010). Product and relationship quiality influence on customer commitment and loyalty in B2B manufacturing relationships. Industrial Marketing Management, v. 39, n. 8, 1321-1333. https://doi.org/10.1016/j.indmarman.2010.02.006

Chaudhuri, A., Holbrook, M. B. (2001). The chain of effects from brand trust and brand affect to brand performance: The role of brand loyalty. Journal of Marketing, v. 65, n. 2, 81-93. https://doi.org/10.1509/jmkg.65.2.81.18255

Chin, W. W. (1998). Commentary: Issues and opinion on structural equation modeling. MIS Quarterly, v. 22, n. 1, VII-XVI. URL

Chiou, J. S., Droge, C. (2006). Service quality, trust, specific asset investment and expertise: direct and indirect effects in satisfaction-loyalty framework. Journal of the Academy of Marketing Science, v. 34, n. 4, 613-627. https://doi.org/10.1177/0092070306286934

Chua, B. -L., Lee, S., Han, H. (2017). Consequences of cruise line involvement: a comparison of first-time and repeat passengers. International Journal of Hospitality Management, v. 29, n. 6, 1658-1683. https://doi.org/10.1108/IJCHM-09-2015-0452

Churchill, G., Surprenant, C. (1982). An Investigation Into the Determinants of Customer Satisfaction. Journal of Marketing Research, v. 19, n. 4 491-504.

https://doi.org/10.1177/002224378201900410

Clark, J. S., Maher, J. K. (2007). If you have their minds, will their bodies follow? Factors effecting customer loyalty in a ski resort setting. Journal of Vacation Marketing, v. 13, n. 1, 59-71. https://doi.org/10.1177/1356766706071209

Clemes, M. D., Gan, C., Ren, M. (2011). Synthesizing the effects of service quality, value, and customer satisfaction on behavioral intentions in the motel industry: An empirical analysis. Journal of Hospitality \& Tourism Research, v. 35, n. 4, 530-568.

https://doi.org/10.1177/1096348010382239 
Crosby, L. A., Evans, K. R., Cowles, D. (1990). Relationship Quality in Services Selling: An Interpersonal Influence Perspective. Journal of Marketing, v. 54, n. 3, 68-81. https://doi.org/10.1177/002224299005400306

Crotts, J. C., Turner, G. B. (1999). Determinants of Intra-Firm Trust in Buyer-Seller Relationships in the International Travel Trade. International Journal of Contemporary Hospitality Management, v. 11, n. 2/3, 116-123. https://doi.org/10.1108/09596119910251020

De Wulf, K., Odekerken-Schröder, G., lacobucci, D. (2001). Investments in consumer relationships: A cross-country and cross-industry exploration. Journal of marketing, v. 65, n. 4, 33-50. https://doi.org/10.1509/jmkg.65.4.33.18386

Dick, A. S., Basu, K. (1994). Customer Loyalty: Toward an Integrated Conceptual Framework. Journal of the Academy of Marketing Science, v. 22, n. 2, 99-113. https://doi.org/10.1177/0092070394222001

Doney, P. M., Cannon, J. P. (1997). An Examination of the Nature of Trust in Buyer-Seller Relationships. Journal of Marketing, v. 61, n. 2, 35-51. https://doi.org/10.1177/002224299706100203

Dorsch, M. J., Swanson, S. R., Kelley, S. W. (1998). The Role of Relationships Quality in the Stratification of Vendors as Perceived by Customers. Journal of the Academy of Marketing Science, v. 26, n. 2, 128-142. https://doi.org/10.1177/0092070398262004

Dwyer, F. R., Schurr, P. H., Oh, S. (1987). Depeloping Buyer-Seller Relationships. Journal of Marketing, v. 51, n. 2, 11-27. https://doi.org/10.2307/1251126

Farooq, M. S., Radovic-Markovic, M. (2016). Modeling entrepreneurial education and entrepreneurial skills as antecedents of intention towards entrepreneurial behavior in single mothers: a PLS-SEM approach. In Radovic-Markovic, M, Kyaruzi, I. S., Nikitovic, Z., Entrepreneurship: Types, Current Trends and Future Perspectives (198-216). ValjevoPrint.

Farooq, M. S., Salam, M., Fayolle, A., Jaafar, N., Ayupp, K. (2018). Impact of service quality on customer satisfaction in Malaysia airlines: A PLS-SEM approach. Journal of Air Transport Management, v. 67, 169-180. https://doi.org/10.1016/i.jairtraman.2017.12.008

Filo, K., Funk, D. C., Alexandris, K. (2008). Exploring the role of brand trust in the relationship between brand associations and brand loyalty in sport and fitness. International Journal of Sport Management and Marketing, v. 3, n. 1-2, 39-57. https://doi.org/10.1504/IJSMM.2008.015960

Fornell, C., Larcker, D. F. (1981). Structural equation models with unobservable variables and measurement error: Algebra and statistics. Journal of Marketing Research, v. 18, n. 3, 382388. https://doi.org/10.2307/3150980 
Fullerton, G. (2003). When does commitment lead to loyalty? Journal of Service Research, v. 5, n. 4, 333-344. https://doi.org/10.1177/1094670503005004005

Ganesan, S. (1994). Determinants of long-term orientation in buyer-seller relationships. Journal of marketing, v. 58, n. 2, 1-19. https://doi.org/10.1177\%2F002224299405800201

Ganesh, J., Arnold, M. J., Reynolds, K. E. (2000). Understanding the Customer Base of Service Providers: an Examination of the Differences between Switchers and Stayers. Journal of Marketing, v. 64, n. 3, 65-87. https://doi.org/10.1509/jmkg.64.3.65.18028

Garbarino, E., Johnson, M. S. (1999). The different roles of satisfaction, trust, and commitment in customer relationships. Journal of Marketing, v. 63, n. 2, 70-87. https://doi.org/10.1177/002224299906300205

Gefen, D., Karahanna, E., Straub, D. W. (2003). Trust and TAM in online shopping: An integrated model. MIS Quarterly, v. 27, n. 1, 51-90. https://doi.org/10.2307/30036519

Gremler, D. D., Van Vaerenbergh, Y., Brüggen, E. C., Gwinner, K. P. (2020). Understanding and managing customer relational benefits in services: a meta-analysis. Journal of the Academy of Marketing Science, v. 48, n. 3, 565-583. https://doi.org/10.1007/s11747-019-00701-6

Grönroos, C. (1997). Value-driven relational marketing: From products to resources and competencies. Journal of marketing management, v. 13, n. 5, 407-419. https://doi.org/10.1080/0267257X.1997.9964482

Gustafsson, A., Jhonson, M. D., Roos, I. (2005). The effects of customer satisfaction, relationship commitment, dimensions and triggers on customer retention. Journal of Marketing, v. 69, n. 4, 210-218. https://doi.org/10.1509/jmkg.2005.69.4.210

Hair, J. F., Hult, G. T. M., Ringle, C., Sarstedt, M. (2017). A primer on partial least squares structural equation modeling (PLS-SEM). Sage Publications.

Han, H., Hyun, S. S. (2018). Role of motivations for luxury cruise traveling, satisfaction, and involvement in building traveler loyalty. International Journal of Hospitality Management, v. 70, 75-84. https://doi.org/10.1016/i.ijhm.2017.10.024

Hess, J., Story, J. (2005). Trust-based commitment: multidimensional consumer-brand relationship. Journal of Consumer Marketing, v. 22, n. 6, 313-322. https://doi.org/10.1108/07363760510623902

Howat, G., Assaker, G. (2013). The hierarchical effects of percieved quality on percieved value, satisfaction and loyalty: Empirical results from public, outdoor aquatic centres in Australia. Sport Management Review, v. 16, n. 3, 268-284. https://doi.org/10.1016/j.smr.2012.10.001 
Hrebiniak, L. G. (1974). Effects of job level and participation on employee attitudes and perceptions of influence. Academy of Management Journal, v. 17, n. 4, 649-662. https://doi.org/10.5465/255644

Hunt, S. D., Arnett, D. B., Madhavaram, S. (2006). The explanatory foundations of relationship marketing theory. Journal of Business, v. 21, n. 2, 72-87. https://doi.org/10.1108/10610420610651296

Hyun, M., Jordan, J. S. (2020). Athletic goal achievement: A critical antecedent of event satisfaction, reparticipation intention, and future exercise intention in participant sport events. Sport Management Review, v. 23, n. 2, 256-270. https://doi.org/10.1016/i.smr.2019.01.007

Ivašković, I. (2019). The stakeholder-strategy relationship in nonprofit basketball clubs. Economic Research-Ekonomska Istraživanja, v. 32, n. 1, 1457-1475. https://doi.org/10.1080/1331677X.2019.1638283

Izogo, E. E., Ogba, I. E. (2015). Service quality, customer satisfaction and loyalty in automovile repair services sector. International Journal of Quality \& Reliability Management, v. 32, n. 3, 250-269. https://doi.org/10.1108/IJQRM-05-2013-0075

Jau-Chuan, K., Ssu-Lang, L., Ming-Yang, K., (2011). Two-unit redundant system with detection delay and imperfect coverage: confidence interval estimation. Quality Technology \& Quantitative Management, v. 8, n. 1, 1-14. https://doi.org/10.1080/16843703.2011.11673242

Khodabandeh, A., Lindh, C. (2020). The importance of brands, commitment, and influencers on purchase intent in the context of online relationships. Australasian Marketing Journal, v. 29, n. 2, 177-186. https://doi.org/10.1016/j.ausmj.2020.03.003

Kotler, P., Keller, K. L. (2006). Marketing Management (12 ${ }^{\text {th }}$ ed.). Edition Pearson Education

Kumar, V., Leone, R. P., Gaskins, J. N. (1995). Aggregate and disaggregate sector forecasting using consumer confidence measures. International Journal of Forecasting, v. 11, n. 3, 361-377. https://doi.org/10.1016/0169-2070(95)00594-2

Lashley, C., Morrison, A. (2003). Hospitality as a'commercial friendship'. Hospitality Review, v. 5, n. 4, 31-36.

Liga Antioqueña de Tenis. (2019). Quiénes somos. URL

Matsuoka, H., Chelladurai, P., Harada, M. (2003). Direct and interaction effects of team identification and satisfaction on intention to attend games. Sport Marketing Quarterly, v. 12 , v. 4, 244-253. 
Melnyk, V., Van Osselaer, S. M., Bijmolt, T. H. (2009). Are women more loyal customers than men? Gender differences in loyalty to firms and individual service providers. Journal of Marketing, v. 73, n. 4, 82-96. https://doi.org/10.1509/jmkg.73.4.082

Mogaji, E., Adeola, O., Hinson, R. E., Nguyen, N. P., Nwoba, A. C., Soetan, T. O. (2021). Marketing bank services to financially vulnerable customers: evidence from an emerging economy. International Journal of Bank Marketing, v. 39, n. 3, 402-428. https://doi.org/10.1108/IJBM-07-2020-0379

Moorman, C., Zaltman, G., Deshpandé, R. (1992). Relationship Between Providers and Users of Market Research: The Dynamics of Trust Within and Between organizations. Journal of Marketing Research, v. 29, n. 3, 314-328. https://doi.org/10.1177/002224379202900303

Moreira, A. C., Silva, P. M. (2015). The trust-commitment challenge in service quality-loyalty relationships. International Journal of Health Care Quality Assurance, v. 28, n. 3, 253-266. https://doi.org/10.1108/IJHCQA-02-2014-0017

Morgan, R., Hunt, S. (1994). The Commitment-Trust Theory of Relationship Marketing. Journal of Marketing, v. 58, n. 3, 20-38. https://doi.org/10.1177/002224299405800302

Murray, D., Howat, G. (2002). The relationships among Service Quality Value, Satisfaction and future intentions of customers at an Australian Sports and Leisure Centre. Sport Management Review, v. 5, n. 1, 25-43. https://doi.org/10.1016/S1441-3523(02)70060-0

Nogales González, J. F. (2006). Estrategias de marketing en clubes deportivos. Revista de Ciencias del Deporte, v. 2, n. 3, 45-52. URL

Nunnally, J.C., Bernstein I.H. (1994). Psychometric theory (3a ed.). McGraw-Hill.

Nuryakin, Ardyan, E. (2018). SMEs' marketing performance: the mediating role of market entry capability. Journal of Research in Marketing and Entrepreneurship, v. 20, n. 2, 122-146. https://doi.org/10.1108/JRME-03-2016-0005

Nyadzayo, M. W., Khajehzadeh, S. (2016). The antecedents of customer loyalty: A moderated mediation model of customer relationship management quality and brand image. Journal of Retailing and Consumer Services, v. 30, 262-270.

https://doi.org/10.1016/i.jretconser.2016.02.002

Oliver, R. L. (1980). A cognitive model of the antecedents and consequences of satisfaction decisions. Journal of marketing research, v. 17, n. 4, 460-469. https://doi.org/10.1177/002224378001700405

Oliver, R. L. (1999). Whence consumer loyalty? Journal of marketing, v. 63, n. 4, suppl1, 33-44. https://doi.org/10.1177/00222429990634s105 
Paek, B., Morse, A., Hutchinson, S., Lim, C. H. (2021). Examining the relationship for sport motives, relationship quality, and sport consumption intention. Sport Management Review, v. 24, n. 2, 322-344. https://doi.org/10.1016/j.smr.2020.04.003

Palmatier, R. W., Scheer, L. K., Houston, M. B., Evans, K. R., Gopalakrishna, S. (2007). Use of relationship marketing programs in building customer-salesperson and customer-firm relationship: differential influences on financial outcomes. International Journal of Research in Marketing, v. 24, n. 3, 210-223. https://doi.org/10.1016/j.ijresmar.2006.12.006

Parasuraman, A., Zeithalm, V. A., Berry, L. L. (1985). A Conceptual Model of Service Quality and its Implications for Future Research. Journal of Marketing, v. 49, n. 4, 41-50. https://doi.org/10.1177/002224298504900403

Payne, A., Frow, P. (2013). Strategic customer management: Integrating relationship marketing and CMR. Cambridge University Press.

Pedragosa, V., Correia, A. (2009). Expectations, satisfaction and loyalty in health and fitness clubs. International journal of sport management and marketing, v. 5, n. 4, 450-464. https://doi.org/10.1504/IJSMM.2009.023371

Pekovic, S., Rolland, S. (2020). Recipes for achieving customer loyalty: A qualitative comparative analysis of the dimensions of customer experience. Journal of Retailing and Consumer Services, v. 56, 102171. https://doi.org/10.1016/j.jretconser.2020.102171

Peña García, G. (2014). El valor percibido y la confianza como antecedentes de la intención de compra online: el caso colombiano. Cuadernos de Administración, v. 30, n. 51, 15-24. URL

Phonthanukitithaworn, C., Sellitto, C. (2018). Perceptions of service quality at football stadiums: influence on fans' intention to attend future games. Managing Sport and Leisure, v. 23, n. 3, 204-224. https://doi.org/10.1080/23750472.2018.1544850

Price, L. L., Arnould, E. J. (1999). Commercial Friendships: Service Provider-Client Relationships in Context. Journal of Marketing, v. 63, n. 4, 38-56. https://doi.org/10.1177/002224299906300405

Priluck Grossman, R. (1998). Developing and managing effective Consumer Relationships. Journal of Product and Brand Managemen, v. 7, n. 1, 27-40. https://doi.org/10.1108/10610429810209719

Quero, M., Ventura, R. (2015). The role of balanced centricity in the Spanish creative industries adopting a crowd-funding organisational model. Journal of Service Theory and Practice, $\mathrm{v}$. 25, n. 2, 122-139. https://doi.org/10.1108/JSTP-09-2013-0182 
Radovic-Markovic, M., Shoaib Farooq, M., Markovic, D. (2017). Strengthening the resilience of small and medium-sized entreprises. In Takács, I. (Ed.), Management, enterprise and benchmarking in the 21st century IV: "Global challenges, local answers" (345-356). Óbuda University. $\underline{U R L}$

Ringle, C. M., Wende, S., Becker, J.-M. (2015). SmartPLS 3. Boenningstedt: SmartPLS GmbH. URL

Schijns, J. M., Caniëls, M. C., Le Conté, J. (2016). The impact of perceived service quality on customer loyalty in sports clubs. International journal of sport management recreation and tourism, v. 24, 43-75. URL

Seyed Javadein, S. R., Khanlari, A., Estiri, M. (2008). Customer loyalty in the sport services industry: the role of service quality, customer satisfaction, commitment and trust. Journal of Human Sciences, v. 5, n. 2. $\underline{U R L}$

Sirdeshmukh, D., Singh, J., Sabol, B. (2002). Consumer trust, value, and loyalty in relational exchanges. Journal of Marketing, v. 66, n. 1, 15-37.

https://doi.org/10.1509/jmkg.66.1.15.18449

Story, J., Hess, J. (2010). Ethical brand management: customer relationship and ethical duties. Journal of Product \& Brand Management, v. 19, n. 4, 240-249. https://doi.org/10.1108/10610421011059568

TaghiPourian, M., Bakhsh, M. (2015). Loyalty: from single-stage loyalty to four-stage loyalty. International Journal of New Technology and Research, v. 1, n. 6, 48-51. URL

Tax, S. S., Brown, S. W., Chandrashekaran, M. (1998). Customer evaluations of service complaint experiences: implications for relationship marketing. Journal of marketing, v. 62, n. 2, 6076. https://doi.org/10.1177/002224299806200205

Taylor, S., Hunter, G. (2003). An exploratory investigation into the antecedents of satisfaction brand attitude and loyalty within the (B2B) eCRM industry. Journal of Consumer Satisfaction, Dissatisfaction and Complaining Behavior, n. 16, 19-35. URL

Vivek, S. D., Gleen Richey Jr, R., Dalela, V. (2009). A longitudinal examination of partnership governance in offshoring: A moving target. Journal of World Business, v. 44, n. 1, 16-30. https://doi.org/10.1016/i.jwb.2008.03.017

Voss, G.B, Parasuraman, A, Grewal, D (1998). The roles of price, performance, and expectations in determining satisfaction in service exchanges. Journal of marketing, v. 62, n. 4, 46-61. https://doi.org/10.1177/002224299806200404

Westbrook, R. A., Oliver, R. L. (1981). Developing better measures of consumer satisfaction: some preliminary results. NA - Advances. En Kent B. Monroe, Ann Abor (Eds. v. 8) Consumer Research (94-99). URL 
Winand, M., Vos, S., Zintz, T., Scheerder, J. (2013). Determinants of service innovation: a typology of sports federations. International Journal of Sport Management and Marketing, v. 13, n. 1-2, 55-73. https://doi.org/10.1504/IJSMM.2013.055194

Wu, H.-C., Ko, Y. J. (2013). Assessment of service quality in the hotel industry. Jorunal of Quality Assurance in Hospitality Tourism, v. 14, n. 3, 218-244. https://doi.org/10.1080/1528008X.2013.802557

Zainudin, M.I., Haji Hasan, F., Othman, A. K. (2019). Halal brand personality and brand loyalty among millennial modest fashion consumers in Malaysia. Journal of Islamic Marketing, v. 11, n. 6, 1277-1293. https://doi.org/10.1108/JIMA-10-2018-0187

Zeithaml, V. A., Bitner, M. J., Gremler, D. D. (2009). Marketing de servicios (5ta Ed.). McGraw-Hill. 